\title{
CombinAd: avaliação e melhoria da prescrição de contracetivos orais combinados na adolescência
}

Andreia Silva e Sousa', Gisela Costa Neves ${ }^{1}$, Afonso Brás Sousa²

\section{RESUMO}

Objetivos: Melhorar e adequar a prescrição de contracetivos orais combinados (COC) às adolescentes da Unidade de Saúde Familiar (USF) do Castelo.

Tipo de estudo: Pré-experimental, pré e pós intervenção, sem grupo controlo.

Local: ACeS Arrábida - USF Castelo.

População: Adolescentes com idades compreendidas entre os 10 e os 18 anos inscritas na USF Castelo e utilizadoras de COC. Métodos: O estudo decorreu através da análise de dados relativamente à dose de estrogénio do COC utilizado e presença ou ausência de contraindicações absolutas, entre fevereiro de 2019 e fevereiro de 2020, o que permitiu classificar as adolescentes em «bem» ou «mal» medicadas. Procedeu-se a uma primeira avaliação em fevereiro de 2019. De seguida, foi realizada uma intervenção que consistiu em duas fases: a primeira, uma sessão clínica na USF dirigida aos médicos e enfermeiros onde foram divulgados os dados pré-intervenção e feita uma breve apresentação teórica acerca da utilização adequada de COC na adolescência; a segunda fase consistiu no fornecimento de material auxiliar de memória em formato de bolso e afixação de cartazes nos gabinetes onde decorrem as consultas de planeamento familiar. Os outcomes definidos foram: uma taxa de melhoria da prescrição de COC, seis meses depois da intervenção, de 20\%; o aumento ou a manutenção da taxa de melhoria inicial, um ano após a intervenção. Foi utilizado o teste exato de Fisher para comparar as taxas de prescrição adequada pré e pós intervenção. Resultados: Atingiu-se a melhoria da prescrição de COC nas adolescentes seis meses após a intervenção (21,6\%), apesar de não ter sido estatisticamente significativa $(p=0,331)$. Contudo, posteriomente, verificou-se duplicação da taxa de prescrições adequadas prévia, com uma taxa de melhoria de $45,6 \%$, estatisticamente significativa $(p<0,001)$.

Conclusão: Os profissionais da USF conseguiram implementar as estratégias e melhorar a taxa de prescrições adequadas de COC nas adolescentes. $O$ envolvimento de toda a equipa que realiza aconselhamento contracetivo poderá ter aumentado a eficácia da intervenção. A aplicação das estratégias em apenas uma unidade limita a generalização dos resultados. Contudo, estratégias simples parecem aumentar a taxa de prescrições adequadas de COC nesta faixa etária.

Palavras-chave: Contracetivos orais combinados;Adolescente; Feminino; Densidade óssea, efeitos de fármacos; Melhoria da qualidade.

\section{INTRODUÇÃO}

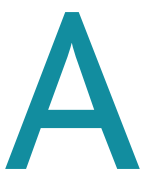
adolescência marca a transição entre a infância e a idade adulta e inclui a faixa etária dos 10 aos 18 anos. ${ }^{1}$ É na adolescência que a maioria dos jovens inicia a atividade sexual. ${ }^{2}$ Os adolescentes são considerados um grupo de interven-

1. Médica de Família. USF Sesimbra, ACeS Arrábida. Sesimbra, Portugal. 2. Médico Interno de Medicina Geral e Familiar. USF Castelo, ACeS Arrábida. Santana, Sesimbra, Portugal. ção prioritária no âmbito da saúde sexual e reprodutiva e na prevenção das infeções sexualmente transmissíveis. Os profissionais de saúde ligados ao aconselhamento contracetivo em adolescentes devem ser competentes na informação prestada sobre os vários métodos de contraceção disponíveis e mais adequados ao grupo etário em questão. ${ }^{3}$

Os adolescentes devem escolher o método contracetivo de acordo com a sua necessidade, expectativa e 
condição médica, promovendo-se a sua responsabilização. ${ }^{3}$

É fundamental na adolescente sexualmente ativa a utilização de métodos contracetivos eficazes de forma correta e consistente, sempre em associação com o preservativo para prevenção simultânea das infeções de transmissão sexual. ${ }^{4}$

A idade por si só não constitui contraindicação à utilização de qualquer método contracetivo. A maioria dos métodos pode ser utilizada sem restrições (classe 1): contraceção hormonal combinada (CHO), progestativo oral (PO) e implante. Os métodos de classe 2 poderão também ser usados, requerendo o seu uso uma vigilância médica específica e são: sistema intra-uterino de levonorgestrel (SIU-LNG), dispositivo intra-uterino de cobre (DIU-Cu), progestativo injetável e método barreira. ${ }^{4}$

A contraceção hormonal combinada (oral, transdérmica, anel vaginal) associa, à elevada eficácia contracetiva, benefícios não contracetivos importantes, como a regularização dos ciclos menstruais, diminuição da dismenorreia, melhoria da acne e prevenção dos quistos funcionais do ovário. ${ }^{4}$

Os progestativos (orais ou implante) são métodos contracetivos de primeira linha nas adolescentes com contraindicação ao uso de estrogénios. ${ }^{2}$ As contraindicações absolutas ao uso de estrogénios que podem estar presentes nestas idades são as seguintes: hemorragia genital não esclarecida; hipertensão arterial não controlada (definida como pressão arterial sistólica $\geq 160 \mathrm{mmHg}$ e diastólica $\geq 90 \mathrm{mmHg}$ ); obesidade mórbida (nesta faixa etária definida como peso acima de $120 \%$ do percentil 95); ${ }^{5}$ trombofilias; história pessoal de tromboembolismo venoso; cirurgia major com imobilização prolongada; doenças cardiovasculares (cardiopatia isquémica, doença valvular complicada) ou cerebrovasculares (acidente vascular cerebral); doença de Raynaud com anticoagulante lúpico positivo; lúpus eritematoso sistémico com anticorpos antifosfolípidos; complicações microvasculares da diabetes (retinopatia/nefropatia/neuropatia); doenças hepatobiliares (tumores hepáticos, hepatites virais, cirrose grave descompensada); enxaqueca com aura; cancro da mama; transplante de órgãos sólidos complicado. ${ }^{3-4}$

Em Portugal, segundo o Inquérito Nacional de Saúde de 2014, os COC são o método contracetivo mais utilizado.
No entanto, alguns estudos suportam a hipótese de que os COC interferem com a aquisição do pico de densidade mineral óssea (DMO) nas adolescentes. ${ }^{6-8}$

A adolescência é um período crítico para aquisição de DMO, sendo que este atingimento é importante para a prevenção da osteoporose. Maximizar o pico de densidade óssea durante a adolescência parece reduzir o risco de fraturas osteoporóticas no futuro. ${ }^{7}$

Na puberdade ocorrem alterações dinâmicas na massa óssea mediadas pela interação entre as hormonas sexuais e a hormona de crescimento (GH). Os estrogénios estimulam a proliferação e diferenciação dos osteoblastos e inibem a sua apoptose, estando também envolvidos na mineralização óssea ao nível da superfície endocortical. Maiores concentrações de estrogénios têm sido associadas a cavidades medulares mais pequenas, maior espessura da cortical e aumento da densidade mineral óssea em adolescentes do sexo feminino. No entanto, os estrogénios exógenos parecem diminuir a responsividade da $\mathrm{GH}$, sendo que nas adolescentes utilizadoras de COC com muito baixas doses de estrogénio ( $15 \mathrm{mcg} / \mathrm{dia})$ tem-se observado um efeito negativo ainda mais acentuado na DMO. ${ }^{6}$

Nas adolescentes, a escolha da dose de estrogénios deve ser individualizada de forma a proteger o metabolismo ósseo. Assim, nestas deve preferir-se dose superior ou igual a $30 \mathrm{mcg}$ por ter menor impacto sobre a DMO (força de recomendação B). ${ }^{3-4,8}$

Na prática clínica habitual das várias listas de utentes da Unidade, os autores notaram que a escolha do método contracetivo das adolescentes tinha particular potencial para ser otimizada.

Face ao exposto, o objetivo do presente estudo foi, durante o período de avaliação de um ano (entre fevereiro de 2019 e fevereiro de 2020), melhorar a prescrição de COC às adolescentes da USF Castelo, assegurando a prescrição de dosagens adequadas de estrogénios às utilizadoras deste método contracetivo e, assim, procurar minimizar os efeitos deletérios sobre a DMO.

\section{MÉTODO}

As USF são pequenas unidades de saúde com autonomia funcional e técnica, que contratualizam objetivos de acessibilidade, adequação, efetividade, eficiência e qualidade e que garantem aos cidadãos inscritos uma carteira básica de serviços. 
A USF Castelo localiza-se num meio rural e é constituída por uma equipa de oito médicos de família, oito enfermeiros e seis administrativos. Esta unidade também é local de formação para internos de formação específica em medicina geral e familiar, internos de formação geral e estudantes de medicina.

As consultas de planeamento familiar (PF) são realizadas pelo médico e pelo enfermeiro de família. Para que a utente possa tomar uma decisão esclarecida é informada acerca das diversas opções disponíveis, do modo de funcionamento e utilização de cada método e das vantagens e desvantagens de cada um, sendo também aconselhada medicamente mediante a sua condição clínica.

Após suspeição da necessidade de adequação dos COC às adolescentes desta unidade foi realizado um estudo quasi-experimental, com avaliação pré e pós intervenção, sem grupo controlo, tendo como outcomes a taxa de melhoria da prescrição de COC nas adolescentes seis meses depois da intervenção e o aumento ou manutenção da taxa de melhoria inicial um ano após a intervenção para avaliar a manutenção dos efeitos desta.

Na população foram incluídas as adolescentes inscritas na USF Castelo com idades compreendidas entre os 10 e 18 anos e utilizadoras de COC. Foram excluídas adolescentes não utilizadoras de qualquer método contracetivo, adolescentes utilizadoras de COC, mas sem registo das dosagens hormonais no processo clínico, e adolescentes utilizadoras de outros métodos contracetivos.

Numa primeira avaliação, a de diagnóstico, em fevereiro de 2019, foram recolhidos dados relativos à idade, método contracetivo e presença ou ausência de contraindicações absolutas, utilizando o programa MedicineOne®.

Seguidamente foi realizada a intervenção. Esta consistiu em duas fases. A primeira, uma sessão clínica na USF dirigida aos médicos e enfermeiros onde foram divulgados os resultados obtidos e uma breve apresentação teórica acerca da utilização adequada dos COC na adolescência, com sensibilização para uma melhor adequação da prescrição destes. Foi definida, por acordo entre os profissionais, uma taxa de $20 \%$ de aumento de prescrições adequadas de COC. A segunda fase consistiu no fornecimento de material auxiliar de me- mória em formato de bolso e afixação de cartazes nos gabinetes onde decorrem as consultas de PF.

A segunda avaliação decorreu em agosto de 2019. Foram recolhidos novamente os dados relativos à idade, método contracetivo e presença ou ausência de contraindicações, utilizando os programas MedicineOne, MIM@UF® e SClinico® (uma vez que o programa MedicineOne ${ }^{\circledR}$ foi descontinuado pela ARSLVT e, consequentemente, na USF Castelo). Foi realizada a avaliação dos resultados com apresentação posterior em reunião de equipa (avaliação da intervenção).

Numa terceira avaliação, em fevereiro de 2020, foi feita uma nova colheita dos dados, utilizando os programas MIM@UF® e SClinico®, e a respetiva avaliação dos resultados (avaliação da manutenção).

Para garantir a confidencialidade e anonimato das utentes, a colheita dos dados foi realizada pela coordenadora da USF Castelo nas três fases do estudo, sendo fornecido à equipa de investigação um ficheiro digital onde constava apenas a data de recolha, a idade ou data de nascimento, utilização ou não de COC, a dosagem utilizada e presença/ausência de contraindicações absolutas ao seu uso. Assegurou-se, assim, que os investigadores apenas tiveram acesso a dados anonimizados.

As variáveis foram codificadas, registadas e tratadas em suporte informático (Microsoft Excel® e IBM SPSS Statistics v. 23®). Através da aplicação do teste exato de Fisher foi feita uma análise comparativa dos resultados obtidos antes e após a intervenção e após a fase de manutenção.

O protocolo do estudo foi aprovado pela Diretora Executiva do ACeS Arrábida, pela coordenadora da USF Castelo e pela Comissão de Ética da Administração Regional de Saúde de Lisboa e Vale do Tejo. O estudo não implicou qualquer custo para o ACeS, não interferiu com o funcionamento regular da unidade nem prejudicou a qualidade dos serviços prestados.

\section{RESULTADOS}

Na primeira avaliação, fase de diagnóstico, foram encontradas 114 adolescentes (Figura 1), das quais 98 cumpriam os critérios de inclusão, com idades compreendidas entre os 13 e os 18 anos. Destas, apenas 35 foram consideradas bem medicadas (Figura 2). De entre as 63 mal medicadas, 57 tinham dose não otimizada e seis tinham contraindicações absolutas (duas com 
enxaqueca com aura registada no processo clínico e quatro com enxaqueca, sem especificação de presença ou ausência de aura, pelo que foi considerado o pior cenário).

Na segunda avaliação, primeira após a intervenção, 96 adolescentes cumpriam os critérios de inclusão (Figura 3), com idades compreendidas entre os 14 e os 18 anos. Destas, 55 foram consideradas bem medicadas e 41 mal medicadas (Figura 2) - 38 por dose não otimizada e três por contraindicações absolutas (uma com enxaqueca com aura e duas com enxaqueca, sem especificação de presença ou ausência de aura). Obteve-se uma taxa de melhoria de $21,6 \%$. No entanto, na comparação da fração de adolescentes bem medicadas dos grupos pré e pós intervenção, através do teste exato de Fisher, não se obteve uma diferença estatisticamente significativa ( $p=0,331$ ).

Na terceira avaliação, segunda após a intervenção, foram incluídas 75 adolescentes (Figura 4) com idades compreendidas entre os $13 \mathrm{e}$ os 18 anos. Destas 61 estavam bem medicadas e 14 mal medicadas (Figura 2), todas por dose não otimizada. Um ano após a intervenção obteve-se uma taxa de melhoria de $45,6 \%$, estatisticamente significativa aplicando o teste exato de Fisher $(p<0,001)$.

\section{DISCUSSÃO}

Apesar de atingida uma melhoria de $21,6 \%$ da prescrição de COC nas adolescentes seis meses após a intervenção, embora superior aos $20 \%$ a que a equipa se propôs, esta melhoria não foi estatisticamente significativa ( $p=0,331$ ). Neste sentido, não se pode concluir

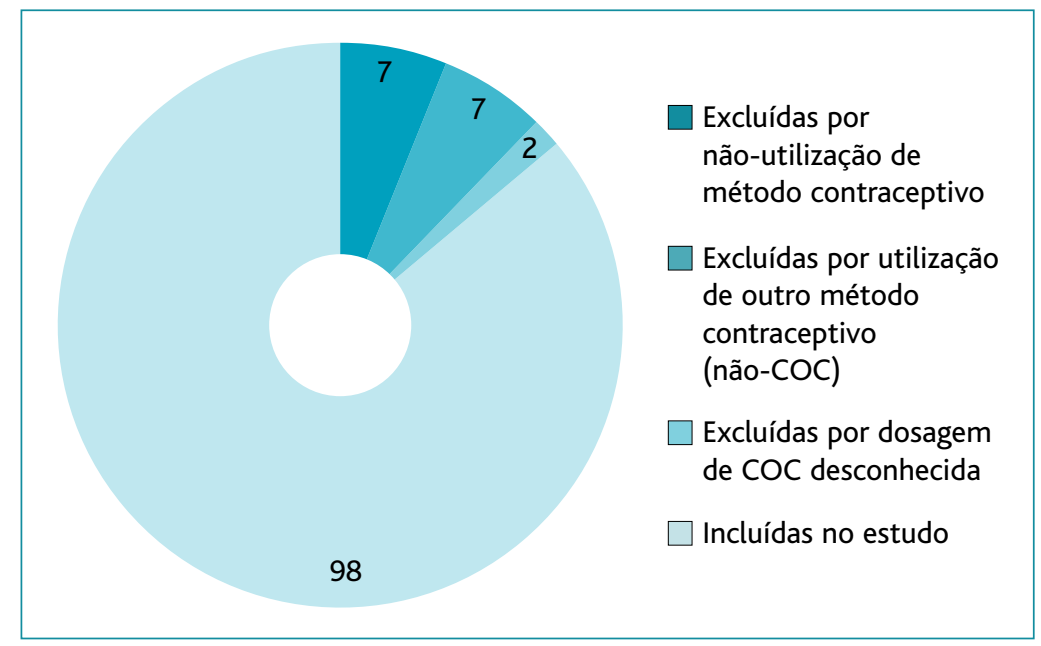

Figura 1. Inclusão/exclusão do estudo (1. a avaliação, fase de diagnóstico).

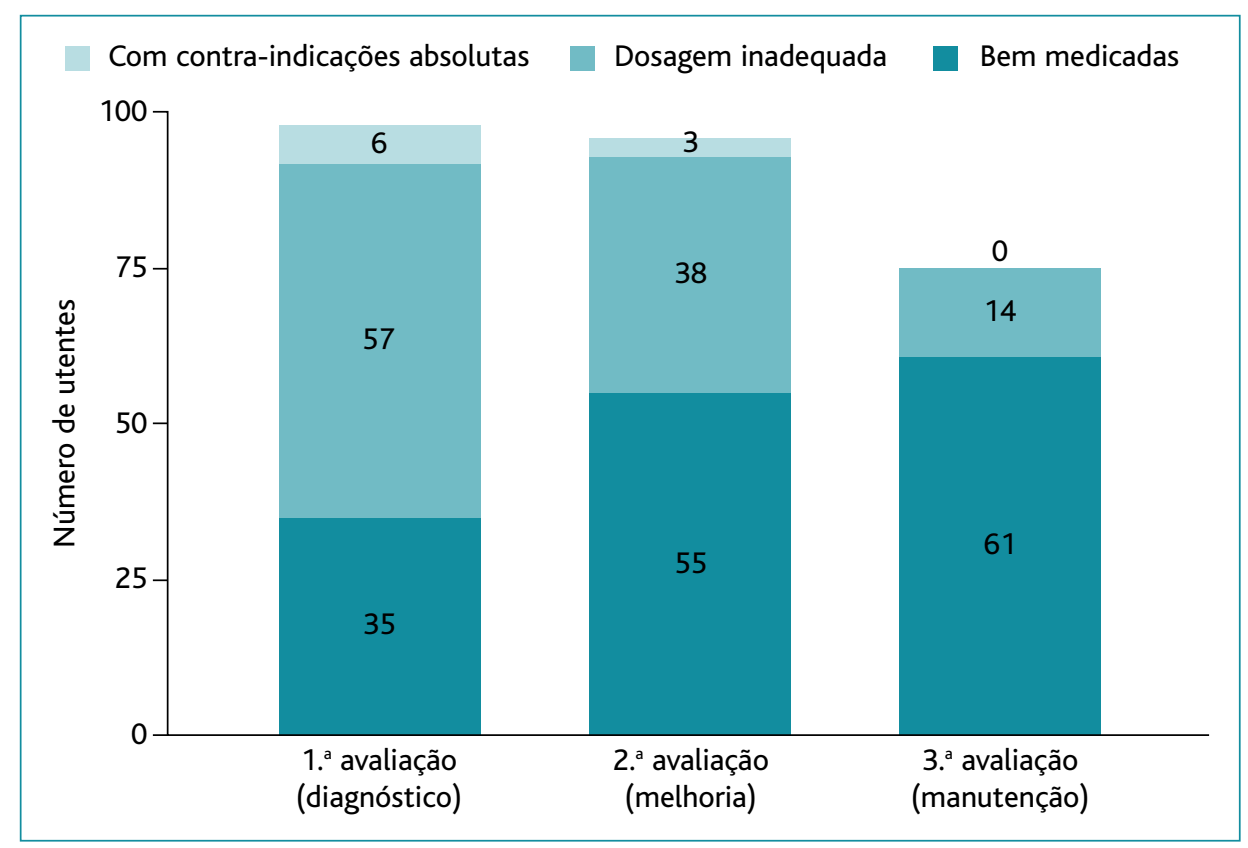

Figura 2. Resultados. que a melhoria, numa primeira fase, se deveu à eficácia da intervenção. Contudo, um ano após a intervenção verificou-se a duplicação da taxa de prescrições adequadas prévia, com taxa de melhoria de $45,6 \%$, estatisticamente significativa $(p<0,001)$.

Estes resultados podem ser explicados pelo facto de a equipa de investigação ter sido demasiado ambiciosa ao querer obter uma melhoria ao fim de apenas seis meses. Por um lado, porque, por norma, esta faixa 


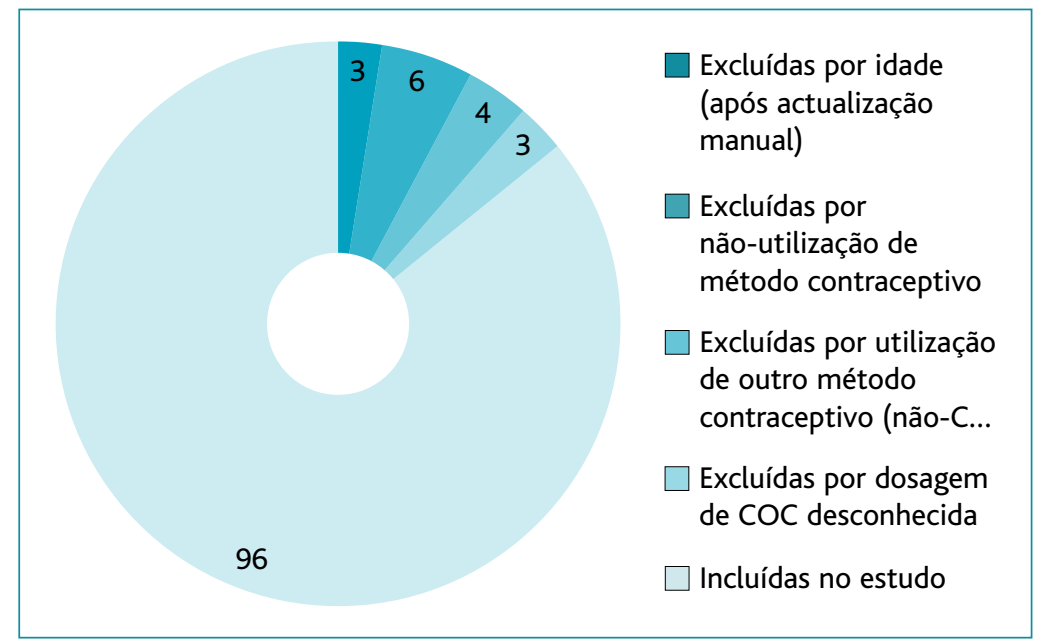

Figura 3. Inclusão/exclusão do estudo (2. avaliação, fase de melhoria).

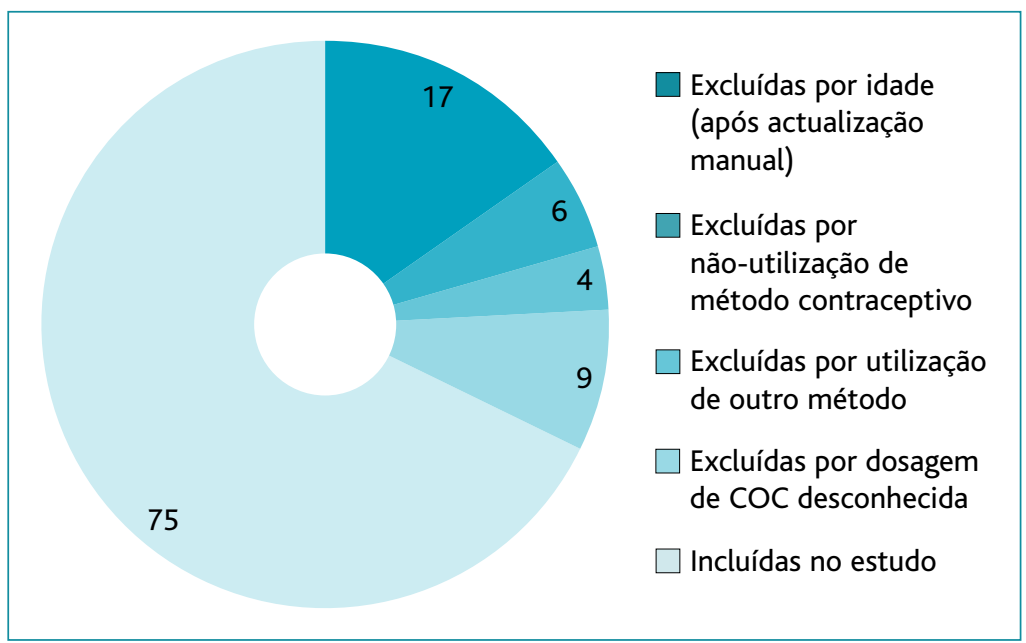

Figura 4. Inclusão/exclusão do estudo (3. avaliação, fase de manutenção).

etária é menos consumidora de cuidados de saúde, o que reduz as oportunidades de mudança de método contracetivo de forma oportunística. Por outro lado, os profissionais de saúde costumam aconselhar uma consulta de planeamento familiar por ano para conseguir dar resposta às necessidades da lista de utentes, motivo pelo qual podem não ser visíveis ao fim de seis meses os esforços exercidos para melhorar.

Outro fator que poderá ter limitado um melhor resultado prende-se com a resistência, quer de mudança de hábitos de prescrição por parte dos profissionais quer por parte das utentes em alterar o método contracetivo.
Para além disso, verificou-se que no fim da terceira avaliação não restava uma única utente com contraindicação absoluta a fazer COC, o que, embora não esteja explicitamente contemplado nos outcomes do estudo, não deixa de ser inequivocamente positivo para a população estudada.

No decorrer do estudo o número de utentes incluídas foi variando substancialmente, algo que poderá ser explicado pelas limitações encontradas ao nível da recolha de dados. Em dezembro de 2018 iniciou-se um processo de transição do sistema informático usado para registo de consultas e processos clínicos - de MedicineOne ${ }^{\circledast}$ para SClinico ${ }^{\circledast}$. Apesar de ter sido efetuada a migração da base de dados dos utentes, esta revelou-se incompleta, com lacunas/conflitos de informação, observados nomeadamente ao nível do programa de planeamento familiar, mais especificamente na contraceção, não se podendo garantir a total correspondência dos dados entre os dois sistemas. Este problema foi-se mitigando com a atualização manual dos dados à medida que os profissionais realizavam novas consultas às utentes. Para além disso, a transição do sistema informático utilizado obrigou à substituição do programa de recolha de dados utilizado, do módulo Estatística incorporado no MedicineOne ${ }^{\circledR}$ para o MIM@UF®.Ambos os programas funcionam de forma bastante diferente e os dados fornecidos por este último tendem a ser mais desatualizados, tendo inclusivamente sido necessária a atualização manual das idades das utentes na recolha de dados efetuada por este último, com exclusão de um número substancial de utentes - 17 (Figura 4). A recolha de dados assenta na informação constante nos processos clínicos das utentes. Apesar de o programa de planeamento familiar ter sido tendencialmente atualizado entre consultas verificou-se que alguns processos não especificaram o COC utilizado. Poderá também haver lacunas noutras áreas, nomeadamente na lista de problemas, que 
poderá não ter o registo codificado de eventuais contraindicações aos COC.

Na literatura não existem estudos equiparáveis. Contudo, o trabalho mais próximo encontrado foi realizado no Reino Unido, na cidade de Bristol, intitulado Improving combined contraceptive pill/oral contraceptives prescribing in general practice, cujo objetivo era adequar a prescrição de COC de acordo com os critérios de elegibilidade da NICE num período temporal de oito meses. No final dos oito meses a adesão aos critérios da Nice foi de $48 \%$, não atingindo os $80 \%$ previstos. ${ }^{9}$ Apesar das diferenças existentes entre o presente estudo e este, os resultados apresentam-se semelhantes. O presente estudo obteve uma melhoria de 45,6\% em 12 meses comparativamente aos $48 \%$ em oito meses deste estudo.

Em Portugal estima-se que a incidência de fraturas de fragilidade da anca seja de 154 a 572 mulheres em cada 100.000 mulheres e de 77 a 232 em cada 100.000 homens, dependendo da idade. A dimensão deste problema tende a aumentar cada vez mais, devido ao envelhecimento progressivo da população e a outras mudanças da sociedade, a menos que sejam adotadas medidas preventivas eficazes. ${ }^{10}$

Muitos fatores de risco para a osteoporose influenciam a saúde dos ossos desde muito cedo e durante toda a vida, mesmo quando as consequências da osteoporose só se tornem aparentes mais tarde na vida. A implementação de medidas de prevenção da osteoporose deve ser aplicada a todas as idades, sempre que sejam identificados fatores de risco corrigíveis. ${ }^{10}$

Maximizar o pico de densidade óssea durante a adolescência parece reduzir o risco de fraturas osteoporóticas no futuro. ${ }^{7}$ Por cada aumento de $10 \%$ da DMO na adolescência, o risco de futuras fraturas diminui $50 \%{ }^{6}$ Daí a importância de, nas adolescentes, se preferir COC com dose superior ou igual a $30 \mathrm{mcg}$ pois, à luz da evidência atual, parece ser uma medida de redução do impacto negativo dos COC sob a DMO.

Apesar do presente estudo apenas incluir uma USF, acredita-se que a intervenção pode ser replicada noutras unidades de saúde com baixas taxas de adequação de COC à faixa etária das adolescentes.

Para os resultados encontrados contribuiu o empenho de toda a equipa e a simplicidade das medidas implementadas.

\section{CONCLUSÃO}

Os profissionais da USF conseguiram implementar as estratégias e melhorar a taxa de prescrições adequadas de COC nas adolescentes, com uma melhoria significativa ao fim de um ano. Acredita-se que o envolvimento de toda a equipa que realiza aconselhamento contracetivo foi fundamental para o aumento da eficácia da intervenção. A aplicação das estratégias em apenas uma unidade limita a generalização dos resultados. Contudo, estratégias simples parecem aumentar a taxa de prescrições adequadas de COC a esta faixa etária.

\section{AGRADECIMENTOS}

À Dra. Lindalva Faria, coordenadora da USF Castelo, pela ajuda imprescindível fornecida e pelo apoio fundamental para a concretização deste projeto.

\section{REFERÊNCIAS BIBLIOGRÁFICAS}

1. Direção-Geral da Saúde. Saúde infantil e juvenil: programa-tipo de actuação. $2^{\mathrm{a}}$ ed. Lisboa: DGS; 2005.

2. Avery L, Lazdane G. What do we know about sexual and reproductive health of adolescents in Europe? Eur J Contracept Reprod Health Care. 2010;15 Suppl 2:S54-66.

3. Neto S, Bombas T, Arriaga C, Almeida MC, Moleiro P. Contraceção na adolescência: recomendações para o aconselhamento contracetivo [Contraception in adolescence: recommendations for counselling].Acta Pediatr Port. 2014;45(1):51-63. Portuguese

4. Sociedade Portuguesa de Ginecologia, Sociedade Portuguesa da Contracepção, Sociedade Portuguesa da Medicina da Reprodução. Consenso sobre contracepção 2011: reunião de consenso nacional sobre contracepção, Estoril, 15 de janeiro de 2011 [Internet]. 2011. Available from: http://www.spdc.pt/files/publicacoes/11_11363_2.pdf

5. Flegal KM, Wei R, Ogden CL, Freedman DS, Johnson CL, Curtin LR. Characterizing extreme values of body mass index-for-age by using the 2000 centers for disease control and prevention growth charts. Am J Clin Nutr. 2009;90(5):1314-20.

6. Cibula D, Skrenkova J, Hill M, Stepan JJ. Low-dose estrogen combined oral contraceptives may negatively influence physiological bone mineral density acquisition during adolescence. Eur J Endocrinol. 2012; 166(6):1003-11.

7. Gai L, Liu X, Gai P, Zhang A, Jiang P, Zhang W. Effect of combined oral contraceptive use on bone mineral density in adolescent females. J Reprod Contracept. 2012;23(3):179-85.

8. Castro JS, Tavares B, Guedes M. Efeito da contraceção com etinilestradiol em alta dose na densidade mineral óssea em adolescentes: qual a evidência? [Effects of ethinylestradiol contraception in bone mineral density in adolescent women: what is the evidence?]. Rev Port Med Geral Fam. 2019;35(4):299-304. Portuguese

9. Russell S, Wiles H. Improving combined contraceptive pill/oral contraceptives prescribing in general practice. BMJ Open Qual. 2017;6(2): e000137. 
10. Marques A, Rodrigues AM, Romeu JC, Ruano A, Barbosa AP, Simões E, et al. Recomendações multidisciplinares portuguesas sobre o pedido de DXA e indicação de tratamento de prevenção das fraturas de fragilidade [Portuguese recommendations for ordering bone densitometry measurement and indications for treatment to prevent osteoporotic fractures]. Rev Port Med Geral Fam. 2016;32(6):425-41. Portuguese

\section{CONFLITO DE INTERESSES}

Os autores declaram não possuir quaisquer conflitos de interesse nem ter obtido financiamentos externos.

\author{
ENDEREÇO PARA CORRESPONDÊNCIA \\ Andreia Silva e Sousa \\ E-mail: andreia_silva_sousa@hotmail.com \\ https://orcid.org/0000-0003-2004-1494
}

Recebido em 30-08-2020

Aceite para publicação em 27-09-2021

\section{ABSTRACT \\ COMBINAD: ASSESSMENT AND IMPROVEMENT OF COMBINED ORAL CONTRACEPTIVES PRESCRIPTION IN ADOLESCENCE}

Objectives: To improve and adapt the prescription of combined oral contraceptives (COC) to adolescents in the Family Health Unit (USF) of Castelo.

Type of study: Pre-experimental, pre, and post-intervention, without a control group.

Place: ACeS Arrábida - USF Castelo.

Population: Adolescents aged 10 to 18 years registered at USF Castelo who use COC.

Methods: The study was carried out by analysing data on the oestrogen dose of the used COC and the presence or absence of absolute contraindications between February 2019 and February 2020, which allowed classifying adolescents as 'well' or 'badly' medicated. A first evaluation was carried out in February 2019. This was followed by an intervention consisting of two phases. The first, a clinical session at the USF for doctors and nurses where the pre-intervention data were disclosed and a brief theoretical presentation on the proper use of COC in adolescence was made. The second phase consisted of the supply of pocket-sized memory aids and the display of posters in the offices where family planning consultations take place. The defined outcomes were: a $20 \%$ improvement rate of the COC prescription, six months after the intervention; an increase or maintenance of the initial improvement rate, one year after the intervention. Fisher's exact test was used to compare the appropriate pre and post-intervention prescription rates.

Results: The improvement of COC prescription was achieved in adolescents six months after the intervention (21.6\%) although it was not statistically significant $(p=0.331)$. However, thereafter, there was a doubling of the rate of previous appropriate prescriptions, with an improvement rate of $45.6 \%$, statistically significant $(p<0.001)$.

Conclusion: USF professionals were able to implement strategies and improve the rate of appropriate COC prescriptions in adolescents. The involvement of the entire contraceptive counselling team may have increased the effectiveness of the intervention. Implementing the strategies in only one unit limits the generalization of results. However, simple strategies appear to increase the rate of appropriate COC prescriptions in this age group.

Keywords: Combined oral contraceptives; Adolescent; Female; Bone density, Drug effects; Quality improvement. 